\title{
Therapeutic vaccines
}

In this age of antimicrobial chemotherapy, the huge amount of work on immunotherapy undertaken before the Second World War has become almost totally forgotten. Sir Almroth Wright, for example, devoted much of his time to the development of therapeutic, as well as prophylactic, vaccines and was portrayed as Sir Bloomfield Bonington in Shaw's play The Doctor's Dilemma with his cry of 'stimulate the phagocytes'. Ironically, it was in Wright's department at St Mary's Hospital, London, that Alexander Fleming discovered penicillin, thereby paving the way to the chemotherapeutic approach to infectious disease.

Once again, however, interest is turning towards the therapy of infectious disease by stimulation of the immune defence mechanisms - for two reasons. First, necessity: there are alarming reports worldwide of drug and multidrug resistance in a wide range of bacterial and protozoal diseases with some microorganisms being resistant to almost all known agents. In the case of tuberculosis, outbreaks of multidrug resistant disease seriously threaten the control of this affliction in many countries a mere two decades after the widespread introduction of short-course chemotherapy.

Secondly, our understanding of the immune system has reached a point at which the design of immunotherapeutic agents, as well as prophylactic vaccines, can be based on rational principles. Although it is well known that harmful hypersensitivity reactions occur in allergic disorders, it is not generally realised that inappropriate immune reactions are responsible for most of the tissue damage and other pathological phenomena in many chronic infectious diseases. Indeed, it is possible to vaccinate an animal in such a way that the outcome of experimental infection is worse than in unvaccinated animals. In tuberculosis, there is ample evidence that the efficacy of Bacille Calmette-Guérin (BCG) varies greatly from region to region; in a large study in South India it appeared to induce a slight predisposition to disease rather than protection [1]. This variation is largely due to previous sensitisation of the population by exposure to environmental mycobacteria [2].

The existence of more than one pattern of reactivity in tuberculosis had been suspected since Koch's pioneering work on Old Tuberculin as an immunotherapeutic agent and has been the subject of considerable controversy [3]. The argument centred on whether the protective immunity and the tissue-destroying reactions represent distinct phenomena or differ only in degree. One problem was that apparently qualitatively different reactions are both transferable by $\mathrm{T}$ cells and both are associated with positive tuberculin reactivity. This subject has become clearer in recent years with the finding that $\mathrm{T}$ cells are not only divisible according to cluster differentiation (CD) antigens but according to their maturation pathways, of which there are two, producing Thl and Th2 helper $\mathrm{T}$ cells which secrete different cytokines [4]. In general, a Thl response is required for protection against chronic intracellular parasitism, as this facilitates detection and lysis of immunologically effete cells packed with pathogens and their containment or destruction by activated macrophages, usually aggregated into granulomas. A Th2 response favours antibody formation and gross tissue necrosis, which may be required for the elimination of very large extracellular parasites, notably worms.

The numerous epitopes of complex pathogens are often regarded as being like the biblical sheep and goats, with some conferring protection and others mediating virulence and immunopathology. The reason for this is largely historical. Most of the early vaccines were for the prevention of acute infections in which protection depends on production of antibody to species- or to strain-specific toxins or factors blocking phagocytosis of the pathogens. However, there is no reason to assume that a 'protective' epitope of an intracellular pathogen such as the tubercle bacillus should have such specificity. Indeed, BCG, an attenuated strain of Mycobacterium bovis, not only protects against tuberculosis, but also against leprosy and lymphadenitis due to environmental mycobacteria, indicating that shared bacterial components are involved in the induction of protection $[1,5]$.

Nevertheless, the dogma of the specific protective antigen is firmly entrenched and much fruitless effort has been spent on isolating single epitopes and studying the nature of the immune response elicited by them. It now appears likely that, rather than a broad spectrum of immune responses being elicited by the complex array of epitopes in a given pathogen, the whole organism elicits a remarkably unified response; a phenomenon termed 'coherence' [6]. It also seems likely that the nature of the coherent response is determined by adjuvants, which have direct effects on the functions of the macrophage and the antigenpresenting cell [7]. 
Adjuvants including peptidoglycans, polyanions, lipoproteins and lipopolysaccharides, have a profound effect on the Th1/Th2 ratio by two principal, and probably closely interacting, mechanisms. One involves the release of cytokines, from macrophages and other cells, that affect the maturation pathway [4]. Thus, for example, interleukin 12 (Il-12) and Il-10 drive, respectively, Th1 and Th2 maturation. Another mechanism involves an effect, directly or via cytokines, on tissue enzymes that convert prohormones into active metabolites which influence the Th1/Th2 ratio. Glucocorticoids, by inhibiting the transcription of Th1 cytokines, favour Th2 maturation while metabolites of dehydroepiandrosterone (DHEA) are antiglucocorticoid and may also directly enhance Thl maturation [8-10].

In the long struggle between pathogens and the mammalian immune system, there would have been a strong evolutionary drive for the latter to select the best possible response to a given pathogen and this strategy usually succeeds. In the case of tuberculosis in immunocompetent persons, only one in 10 of those infected eventually develops clinically evident disease. As the bacillus must be eliciting a highly successful response in the other nine, it is necessary to ask what is different in those who do not control or, contain the infection.

T cell maturation pathways tend to be 'locked in' to one or other type but there are mechanisms for a Th1 to Th2 switch in chronic infections, with detriment to the host [6]. These include changes in the glucocorticoid/DHEA ratio due to effects of cytokines and disease-related stress on the hypothalamo-pituitaryadrenal axis and a direct effect of circulating tumour necrosis factor on the adrenal gland [9].

In tuberculosis, a Thl response favours protection while a Th2 (or mixed Th1 and Th2) response causes the massive and inappropriate tissue destruction responsible for the formation of lung cavities in which the tubercle bacilli may freely replicate and gain access to the cough spray, thereby infecting other persons [11]. Accordingly, a shift towards Thl responsiveness should be beneficial. This may be achieved with a strain of $M$. vaccae which not only contains the adjuvant compound or compounds, but also the common mycobacterial antigens [12]. A heatkilled suspension of this organism is effective against drug-susceptible and multidrug-resistant tuberculosis when used as an adjunct to antimicrobial chemotherapy $[13,14]$.

Can $M$. vaccae be used an an immunotherapeutic agent for other chronic infectious diseases, for example, by introducing epitopes of unrelated pathogens by genetic recombination [15]? In fact, there is anecdotal evidence that $M$. vaccae is effective alone in unrelated infections, perhaps by recognition of markers of stress on infected cells rather than pathogenspecific antigen. All cells contain small amounts of 'heat-shock proteins' (HSP), most of which are normally involved in the folding and assembly of newly synthesised proteins. In circumstances of stress, such as heat shock or intracellular parasitism, the quantity of HSPs increases greatly and they are presented on the cell surface where they serve as targets for 'hunter-killer' cells [16].

Patients with tuberculosis and leprosy have a diminished ability to respond to the common mycobacterial antigens $[17,18]$, as have tuberculin positive HIVinfected persons, patients with rheumatoid arthritis and persons in the silent phase of Chagas' disease (South American trypanosomiasis) $[19,20]$. These common antigens include HSPs.

The modulation of immune recognition of HSPs by bacterial adjuvants suggests that immunotherapeutic agents may have a place in the treatment of autoimmune disease and cancer. HSPs are highly conserved so that one from a mycobacterium is structurally and antigenically similar to its human analogue. One of the paradoxes of immunology is that the conserved HSPs are immunodominant and thus, whether of exogenous or endogenous origin, are potentially capable of inducing 'anti-self' or autoimmune responses. Normally this is controlled by a complex immunoregulatory network but the high incidence of autoimmune disease indicates that this network can easily malfunction [16]. It seems that $M$. vaccae immunotherapy may, in some circumstances, correct such a malfunction, as has been demonstrated in psoriasis and psoriatic arthropathy [21].

Another group of diseases involving Th2 cytokines and IgE are the atopic or allergic conditions such as urticaria, hay fevers and extrinsic asthma [22]. An obvious therapeutic strategy is to incorporate the relevant epitope of an allergen into a Th1 adjuvant by genetic recombination or chemical conjugation.

Bacterial products have long been used as therapeutic agents in cancer. The first of these were the 'Coley toxins', extracts of streptococci and Serratia species, which showed some efficacy against sarcomas. Neonatal BCG vaccination also confers considerable protection against leukaemia and other childhood cancers [23]. It has also been used in the treatment of cancers, either alone or as a carrier for tumour antigens, although with variable results and frequent serious side effects. It is probable that the efficacy of BCG, and the severity of side effects, depend on whether there is a predetermined Th1 or Th2 response and that more consistent results, with minimal side effects, would be obtained by use of $M$. vaccae. This reasoning is based on the hypothesis that an important component of antitumour immunity is recognition and cytolysis of stressed cancer cells expressing HSPs on 
their surface [24]. Thus, murine tumour cell lines transfected with, and expressing, a mycobacterial 65kDa HSP lost their ability to cause tumours and induced a cytotoxic immunity to the analogous untransfected cancer cells [25]. Preliminary studies of $M$. vaccae immunotherapy in cancer patients are being conducted.

It is evident that therapeutic vaccines with Th1 adjuvant activities are likely to act in several different ways. First, they down-regulate inappropriate Th2driven responses that cause immunopathology in a range of chronic infections characterised by intracellular parasitism. Secondly, they induce a Th1 response leading to macrophage activation and granuloma formation. Thirdly, they appear to facilitate a regulated recognition of HSPs thereby suppressing autoimmune phenomena, but also facilitating the recognition of stressed, abnormal cells expressing HSPs on their surfaces. Fourthly, they reduce Th2-mediated allergic responses. Thus they have the potential to treat chronic infections, autoimmune disease, cancer and allergy, which make up a very large part of the ills that afflict mankind. Finally, there is increasing evidence that vasculitis, which is an important component in the immunopathology of many diseases and a causative factor in arteriosclerosis, is triggered by Th2 cytokines and thus amenable to immunotherapeutic intervention. Although Thl activity appears beneficial in a wide range of disorders, there are circumstances in which this may not be desirable. Thus down-regulation of Th1 activity without stimulating Th2-driven antibody formation is likely to prolong homograft survival [7], while a specific Th2 response may be required for protection against helminth infection.

The wide range of activity of adjuvants, notably those driving Th1 responses, with or without the presence or addition of specific antigen, suggests that these agents may lead to as significant and far-reaching a therapeutic revolution in the 21 st century as antimicrobial drugs did in the 20th century.

\section{J. M. GRANGE and J. L. STANFORD National Heart and Lung Institute, Imperial College, London and Department of Bacteriology, University College London Medical School, London.}

\section{References}

1. Fine PEM. Variation in protection by BCG: implications of and for heterologous immunity. Lancet 1995; 346: 1339-1345.

2. Stanford JL, Shield MJ, Rook GAW. How environmental mycobacteria may predetermine the protective efficacy of BCG. Tubercle 1981; 62: 55-62.
3. Bothamley GH, Grange JM. The Koch phenomenon and delayed hypersensitivity 1891-1991. Tubercle 1991; 72: 7-12.

4. Mosmann TR, Moore KW. The role of Il-10 in crossregulation of Th1 and Th2 responses. Immunol Today 1991; 12: A49A53.

5. Trnka L, Dankova D, Svandova E. Six years' experience with the discontinuation of BCG vaccination. 4. Protective effect of BCG vaccination against the Mycobacterium avium intracellulare complex. Tubercle Lung Dis 1994; 75: 348-352.

6. Bretscher PA, Wei G, Menon JN, Bielefeldt-Ohmann $H$. Establishment of stable cell-mediated immunity that makes 'susceptible' mice resistant to Leishmania major. Science 1992; 257: 539-542.

7. Rook GAW, Stanford JL. Adjuvants, endocrines and conserved epitopes; factors to consider when designing 'therapeutic vaccines'. Int J Immunopharmacol 1995; 17: 91-102.

8. Daynes RA, Araneo BA, Hennebold J, Enioutina E, Mu HH. Steroids as regulators of the immune response. $J$ Invest Dermatol 1995; 105 Suppl: 14S-19S.

9. Baker R, Zumla A, Rook GAW. Tuberculosis, steroid metabolism and immunity. $Q J$ Med 1996; 89: 387-394.

10. Rook GAW, Honour J, Kon OM, Wilkinson RJ, Davidson R, Shaw RJ. Urinary adrenal steroid metabolites in tuberculosis; a new clue to pathogenesis? $Q J$ Med 1996; 89: 333-341.

11. Filley EA, Bull HA, Dowd PM, Rook GAW. The effect of Mycobacterium tuberculosis on the susceptibility of human cells to the stimulatory and toxic effects of tumour necrosis factor. Immunology 1992; 77: 505-509.

12. Hernandez-Pando R, Rook GAW. The role of TNF $\alpha$ in T cellmediated inflammation depends on the Th1/Th2 cytokine balance. Immunology 1994; 82: 591-595.

13. Stanford JL, Stanford CA, Rook GAW, Grange JM. Immunotherapy for tuberculosis. Investigative and practical aspects. Clin Immunother 1994; 1: 430-440.

14. Stanford JL, Stanford CA. Immunotherapy for tuberculosis with $M$. vaccae. J Med Microbiol 1996; 44: 24-34.

15. Garbe RT, Barathi J, Barnini S et al. Transformation of mycobacterial species using hygromycin resistance as selectable marker. Microbiology 1994; 140: 133-138.

16. Cohen IR, Young DB. Autoimmunity, microbial immunity and the immunological homunculus. Immunol Today 1991; 12: $105-110$.

17. Stanford JL, Nye PM, Rook GAW, Samuel N, Fairbank A. A preliminary investigation of the responsiveness or otherwise of patients and staff of a leprosy hospital to groups of shared and species antigens of mycobacteria. Lepr Rev 1981; 52: 321-327.

18. Kardjito T, Beck JS, Grange JM, Stanford JL. A comparison of the responsiveness to four new tuberculins among Indonesian patients with pulmonary tuberculosis and healthy subjects. Eur $J$ Respir Dis 1986; 69: 142-145.

19. Khoo SH, Wilkins EGL, Fraser I, Stanford JL. Lack of T cell response to common mycobacterial antigens in HIV-infected individuals - consequence or co-factor? IX International conference on AIDS, Berlin, 6-11 June, 1993. Abstract POA24-0551.

20. Bottasso $\mathrm{OA}$, Ingledew $\mathrm{N}$, Keni $\mathrm{M}$ et al. Cellular immune response to common mycobacterial antigens in subjects seropositive for Trypanosoma cruzi. Lancet 1994; 344: 1540-1541.

21. Ramu G, Prema GD, Balakrishnan S, Shankar Narayan NP, Stanford JL. A preliminary report on the immunotherapy of psoriasis. Ind Med Gaz 1990; 124: 381-382.

22. Martinez FD. Role of viral infections in the inception of asthma and allergies during childhood: could they be protective? Thorax 1994; 49: 1189-1191.

23. Grange JM, Stanford JL. BCG vaccination and cancer. Tubercle 1990; 71: 61-64.

24. Grange JM, Stanford JL, Rook GAW. Tuberculosis and cancer: parallels in host responses and therapeutic approaches? Lancet 1995; 345: 1350-1352.

25. Lukacs KV, Lowrie DB, Stokes RW, Colston MJ. Tumor cells transfected with a bacterial heat-shock gene lose tumorigenicity and induce protection against tumors. J Exp Med 1993; 178: $343-348$. 